\title{
Article \\ Determinants and Dynamic Changes of Generic Quality of Life in Human Bladder Cancer Patients
}

\author{
Yuh-Shyan Tsai ${ }^{1, *}++^{\mathbb{D}}$, Tzu-Yi Wu ${ }^{2,+} \mathbb{D}$, Yeong-Chin Jou ${ }^{3,4,+}$, Tzong-Shin Tzai ${ }^{5}$ and Jung-Der Wang ${ }^{6,7} \mathbb{D}$ \\ 1 Department of Urology, National Cheng Kung University Hospital, College of Medicine, National Cheng \\ Kung University, Tainan 704, Taiwan \\ 2 Department of Occupational Therapy, Asia University, Taichung 413, Taiwan; tywu820@asia.edu.tw \\ 3 Department of Urology, Ditmanson Medical Foundation, Chiayi Christian Hospital, Chia-Yi 60002, Taiwan; \\ 01729@cych.org.tw \\ 4 Department of Food Nutrition and Health Biotechnology, Asia University, Taichung 413, Taiwan \\ 5 Department of Urology, Tainan Municipal An-Nan Hospital, Tainan 709, Taiwan; tts777@gmail.com \\ 6 Departments of Internal Medicine and Occupational and Environmental Medicine, National Cheng Kung \\ University Hospital, Tainan 704, Taiwan; jdwang121@gmail.com \\ 7 Department of Public Health, College of Medicine, National Cheng Kung University, Tainan 701, Taiwan \\ * Correspondence: youh@mail.ncku.edu.tw; Tel.: +886-6-2353535-5251; Fax: +886-6-2383678 \\ + Tsai, Y.-S; Wu, T.-Y. and Jou, Y.-C. contribute equally to this article.
}

Citation: Tsai, Y.-S.; Wu, T.-Y.; Jou,

Y.-C.; Tzai, T.-S.; Wang, J.-D.

Determinants and Dynamic Changes of Generic Quality of Life in Human Bladder Cancer Patients. J. Clin. Med. 2021, 10, 5472. https://doi.org/ $10.3390 / \mathrm{jcm} 10235472$

Academic Editor: Marco Roscigno

Received: 11 October 2021

Accepted: 17 November 2021

Published: 23 November 2021

Publisher's Note: MDPI stays neutral with regard to jurisdictional claims in published maps and institutional affiliations.

Copyright: (C) 2021 by the authors Licensee MDPI, Basel, Switzerland. This article is an open access article distributed under the terms and conditions of the Creative Commons Attribution (CC BY) license (https:// creativecommons.org/licenses/by/ $4.0 /)$

\begin{abstract}
We measured and determined the factors associated with long-term generic quality-of-life (QOL) changes in human bladder cancer patients. We utilized the World Health Organization QOLBrief questionnaire to assess consecutive patients' QOL at outpatient clinics of our hospital. A mixedeffects model was constructed to investigate the determinants of QOL changes according to each domain and individual item after controlling for demographic and clinical factors, as well as the effect of radical cystectomy. We also applied a kernel smoothing method to describe the long-term dynamic changes after the first definite treatment. In total, 1185 repeated measurements were collected from 343 bladder cancer patients. The mixed-effects models demonstrated that marital status, monthly income, and comorbidity with heart disease and diabetes were significant determinants among all the study participants. Regardless of the urinary diversion type, radical cystectomy contributed to lower scores for all four domains, mainly from 4-5 years after cystectomy, which declined significantly in patients who were older than 60 years. As for non-muscle-invasive bladder cancer (NMIBC) patients with preserved bladders, tumor recurrence was a major predictor for lower scores for sexual activity in the social domain. In summary, generic QOL can be independently influenced by many factors, not only cystectomy and tumor recurrence, which should be discussed with patients before treatment.
\end{abstract}

Keywords: quality of life; bladder cancer; dynamic changes; cystectomy

\section{Introduction}

Bladder cancer is the ninth most frequently diagnosed malignancy in the world, and high-incidence areas include Southern Europe, North America, Northern Africa, and Western Asia [1]. There is a high incidence of bladder cancer in the blackfoot endemic area located on the southwest coast of Taiwan due to chronic arsenicism [2,3]. About 70-80\% of bladder cancer cases are diagnosed as non-muscle-invasive bladder cancer (NMIBC). Most of these (about 70\%) readily recur after endoscopic resection, and about $15 \%$ progress to muscle-invasive status even under regular cystoscopic surveillance, adjuvant intravesical chemotherapy, or intravesical Bacillus Calmette-Guérin (BCG) immunotherapy [4]. High-risk NMIBC has a higher probability of developing into BCG-resistant tumors and progressing to muscle-invasive bladder cancer (MIBC), which requires a radical cystetomy, or more severe conditions. In contrast, $20-30 \%$ of bladder cancer cases are initially muscle-invasive, advanced, or metastatic tumors. Currently, the standard therapy for muscle-invasive bladder cancer (MIBC) is a radical cystectomy with various types of 
urinary diversion or reservoir. As for those patients who are unwilling or unfit for a radical cystectomy, a bladder-sparing strategy involving the maximal transurethral resection of the bladder tumor (TURBT) or a partial cystectomy plus either adjuvant systemic chemotherapy, radiation, or both is the alternative approach [5]. Systemic chemotherapy remains the mainstream frontline therapy for metastatic diseases, since immune checkpoint inhibitors remain in their infancy [6]. Moreover, the combination of chemotherapy and immunotherapy fails to show any statistically significant success [7]. Owing to the limited success of the neoadjuvant use of such a promising immunotherapy in MIBC patients before cystectomy [8], a radical cystectomy remains burdensome to most patients and urologists and requires more investigation and surveillance [9].

Many studies have reported on the QOL of bladder cancer patients, but the majority are cross-sectional and may not be applicable for reflecting long-term effects [10-19]. In fact, some of these focus specifically on the quality of life of patients with a urinary diversion [4], and some of these are not adjusted for confounding variables [20]. Moreover, there are many unmet needs in terms of supportive care for bladder cancer patients. In addition, quality of life often differs across disease groups and cancer survivorship trajectories [21]. Thus, it is necessary to investigate the determinants and dynamic changes in the QOL of human bladder cancer patients using a generic questionnaire with a longitudinal follow-up, after ensuring the control of potential confounders and comorbidities.

To promote our understanding of and precision in dealing with bladder cancer patients, the aims of this study were to explore the factors associated with QOL changes after controlling for potential confounding comorbidities and to explore the long-term dynamic changes in QOL among bladder cancer patients who did and did not receive a cystectomy.

\section{Materials and Methods}

\subsection{Participants}

This study began after the approval of the Institutional Review Board was obtained, in 2013 (NCKUH A-ER-101-219). We collected the results of self-reported QOL questionnaires filled out by bladder cancer patients who had provided consent before an outpatient visit. We enrolled patients who were at any clinical stage or were receiving any treatment option to participate, without any exclusion criteria. The treatment strategies were based on the bladder cancer treatment guidelines of the NCKUH modified from the NCCN guidelines. The participants' QOL was measured repeatedly using the QOL questionnaire during subsequent visits and follow-ups.

\subsection{Measurements of $Q O L$}

The abbreviated World Health Organization Quality of Life (WHOQOL-BREF) questionnaire [22] was used for measurements of QOL and contains four domains (comprising 26 items) - physical, psychological, social, and environmental. All items are rated on a 5-point Likert scale. A higher score represents a better quality of life. The domain scores are calculated by multiplying the mean score of the items in the domain by four. Thus, the score of each domain ranges from 4 to 20 . With two additional locally specific items (being respected and eating), the Taiwanese version of the WHOQOL-BREF has been validated to exhibit a good test-retest reliability (correlation coefficient $>0.75$ ), good internal consistency reliability (Cronbach's $\alpha>0.91$ ), and good construct validity in patients with several malignancies [23-25].

\subsection{Procedures}

The participants were instructed on how to self-complete the Taiwanese version of the WHOQOL-BREF questionnaire through tablet computers. If any questions arose about the definition of a query or item, an experienced research assistant was available to clarify the meaning of the items in a standardized way. We also collected the patients' information, including demographic (age at time of investigation, gender, marital status, education level, and monthly family income) and clinical information (age at diagnosis, clinical 
or pathological stage, date and types of surgery, periods of systemic chemotherapy and radiotherapy). The former was collected via person-to-person interviews for illiterate patients. The latter was abstracted from the electronic medical records of the hospital.

\subsection{Statistical Analysis}

The participants were stratified into two categories for analysis: those with NMIBC and those with MIBC or more severe conditions. A kernel-type smoother was utilized to illustrate the dynamic changes of each item and the domain scores via the open access software, iSQOL $[25,26]$. The timeline of the dynamic changes (duration to date for each measurement/interview) was measured in months since the diagnosis of bladder cancer. The trends in the QOL changes were compared between those patients with and without a cystectomy. Mixed-effects models were utilized to find the determinants of generic QOL while controlling for potential confounding factors, including demographic and clinical parameters. These parameters included age at the time of the measurements (i.e., $\geq 70$ years old, 60-69 years old, and $<60$ years old), gender, marital status, education level, monthly family income (i.e., more or less than USD 1750), comorbidities, disease stage, whether or not a cystectomy had been performed, time since chemotherapy (i.e., more or less than 12 months since the first chemotherapy), the history of definite or salvage radiotherapy (i.e., yes or no), and the interaction between age groups and radical cystectomy. The mixed-effects models were analyzed using the IBM SPSS 20 software.

\section{Results}

\subsection{Demographics and Clinical Characteristics of the Participants}

A total of 343 bladder cancer patients completed the WHOQOL-BREF, with a sum of 1185 repeated measurements. Fifty-two (15\%) patients underwent radical cystectomy (radical cystectomy + neobladder, 19; radical cystectomy + ileal conduit, 25; radical cystectomy + ileal reservoir with the Mitrofanoff procedure, 4; radical cystectomy alone, 4). Twenty-nine patients died during follow-up. Eighty-one patients (23.6\%) completed the WHOQOL-BREF once only, and these patients had a higher mortality rate and rate of heart disease compared with those who completed the WHOQOL-BREF more than once (mortality rate, $28.4 \%$ vs. $2.3 \%$; proportion of patients with heart disease, $19.8 \%$ vs. $8.8 \%$ ) (Table S1). The mean age of all the participants at the time of the interview was $67.1 \pm 11.4$ years, and $238(69.4 \%)$ of them were male. Table 1 summarizes the demographic and clinical characteristics of the participants. In total, 250 of $343(72.9 \%)$ were married or cohabiting, and there was a higher frequency of such statuses among cystectomized patients compared with those who had not undergone a cystectomy $(p=0.04)$. There were no significant differences in terms of age, years in education, and monthly family income between patients who had and had not undergone a cystectomy $(p>0.05)$. Moreover, those who had undergone a cystectomy were less often comorbid with diabetes mellitus than those who had not $(5.8 \%$ versus $19.9 \%, p=0.01)$. The median length of the period from bladder cancer diagnosis to the participants' first measurements was 14.6 months. The proportion of patients who completed more than one assessment for both groups (those who received bladder-sparing treatment versus cystectomized patients) in each period can be found in Table S1.

\subsection{Determinants of Generic QOL Using Mixed-Effects Models}

Because only $10(4 \%)$ of the 234 NMIBC patients received a cystectomy, we analyzed the significance of the clinical and demographic factors for the score of each item of the WHOQOL-BREF based on the 224 patients who did not receive a cystectomy. Table 2 shows the results analyzed using the mixed-effects-model method. Both being married/cohabiting and having spent longer in education were the most significant factors for the enhancement of participants' QOL in the majority of items within the four domains. Male patients had higher scores for certain items in the social and environment domains, such as being respected, physical environment, and financial resources. Patients with a monthly family 
income greater than USD 1750 had higher scores in several items within the environment domain than those with a lower income did. Comorbidity either with heart disease, diabetes, or other malignancies reduced the QOL score for the physical domain's medication item. Patients with bladder tumor recurrence had lower scores for the psychological domain's positive feelings item and the social domain's sexual activity item (Table 2).

Table 1. Demographic and clinical characteristics of bladder cancer patients.

\begin{tabular}{|c|c|c|c|c|}
\hline \multirow{2}{*}{ Parameters } & \multirow{2}{*}{ All } & \multicolumn{2}{|c|}{ Cystectomy } & \multirow{2}{*}{$p$ Value } \\
\hline & & No & Yes & \\
\hline Total no. of patients & 343 & 291 & 52 & \\
\hline Total no. of measurements & 1185 & 963 & 222 & \\
\hline Measurements per person & 3.4 & 3.3 & 4.2 & \\
\hline Age (years); mean \pm SD & $67.0 \pm 10.5$ & $67.0 \pm 11.8$ & $67.7 \pm 9.4$ & 0.61 \\
\hline Age group & & & & 0.19 \\
\hline$\geq 70 \mathrm{y} / \mathrm{o}$ & 144 & 123 & 21 & \\
\hline $60-69$ y/o & 99 & 79 & 20 & \\
\hline$<60 \mathrm{y} / \mathrm{o}$ & 100 & 89 & 11 & \\
\hline Gender (male/female) & $238 / 105$ & $192 / 88$ & $46 / 17$ & 0.59 \\
\hline Disease status $(\mathrm{N})$ & & & & $<0.0001$ \\
\hline NMIBC & 234 & 224 & 10 & \\
\hline MIBC or more severe condition & 109 & 67 & 42 & \\
\hline Measurements per person & & & & Not assessed \\
\hline NMIBC & 3.4 & 3.4 & 3.6 & \\
\hline MIBC or more severe condition & 3.6 & 3.1 & 4.0 & \\
\hline Education (years) & $9.0 \pm 4.7$ & $9.0 \pm 4.8$ & $9.1 \pm 4.6$ & 0.93 \\
\hline $\begin{array}{l}\text { Marital status (married or } \\
\text { cohabiting/other) }\end{array}$ & $250 / 93$ & $218 / 73$ & $32 / 20$ & 0.04 \\
\hline $\begin{array}{l}\text { Monthly family income }>\text { USD } \\
1750 \text { (yes/no/missing) }\end{array}$ & $106 / 229 / 7$ & $94 / 192 / 5$ & $12 / 37 / 2$ & 0.24 \\
\hline $\begin{array}{l}\text { Months since first treatment } \\
\text { (median, quartile deviation) }\end{array}$ & $14.6(30.0)$ & $14.5(30.3)$ & $14.9(27.5)$ & - \\
\hline Comorbidity & & & & \\
\hline Diabetes mellitus & $61(17.8 \%)$ & $58(19.9 \%)$ & $3(5.8 \%)$ & 0.01 \\
\hline Heart Disease & $39(11.4 \%)$ & $37(12.7 \%)$ & $2(3.8 \%)$ & 0.06 \\
\hline Other malignancies & $12(3.5 \%)$ & $7(2.4 \%)$ & $5(9.6 \%)$ & 0.01 \\
\hline $\begin{array}{l}\text { Systemic chemotherapy in the } \\
\text { past year }\end{array}$ & $17(5.0 \%)$ & $13(4.5 \%)$ & $4(7.7 \%)$ & 0.32 \\
\hline Radiotherapy & $11(3.2 \%)$ & $10(3.4 \%)$ & $1(1.9 \%)$ & 0.57 \\
\hline
\end{tabular}

NMIBC, non-muscle-invasive bladder cancer; MIBC, muscle-invasive bladder cancer; SD, standard deviation; USD, United States dollar.

In total, $52(15 \%)$ of the 343 patients received a radical cystectomy; we subsequently analyzed the influence of the radical cystectomy on the score for each item of the WHOQOLBREF in all study subjects, as well as the influence of the clinical and demographic factors. Table 3 shows the results analyzed using the mixed-effects-model method. As previously, both being married/cohabiting and having a higher monthly family income were the most significant factors for the enhancement of the participants' QOL for every item in the four domains, as well as comorbidity with heart disease. Diabetes comorbidity reduced the QOL scores for the three items in the physical domain, including medication, energy and fatigue, and mobility. Patients with a muscle-invasive bladder tumor or a more severe condition had lower scores for several items in the physical, psychological, and environment domains. Patients who received a radical cystectomy scored lower for the sexual activity item in the social domain. Interestingly, such a negative effect of a radical cystectomy on sexual activity was less apparent in patients aged 60-69 years and $\geq 70$ years compared with those aged less than 60 years (Table 3 ). 


\subsection{Dynamic Changes in QOL with Radical Cystectomy}

\subsubsection{Domain Score}

As for all the study subjects, the cystectomized patients in the subgroup of patients with MIBC or more severe conditions had lower QOL scores for all four domains, particularly 5 years after their initial definite treatment, compared with patients who received bladder-sparing treatment. Moreover, there was a significant decline in the score for the social domain in NIMBC patients who received a cystectomy compared with those who were treated via a bladder-sparing strategy (Figure 1).

(A)

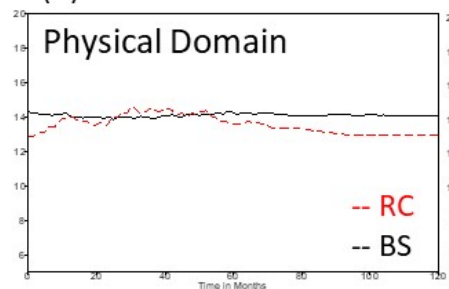

(E)

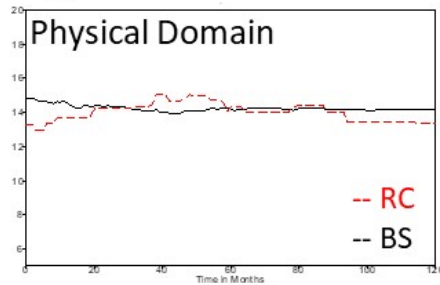

(I)

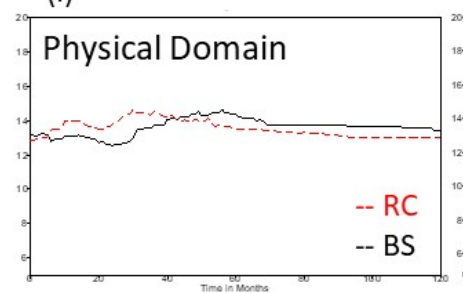

(B) (C)

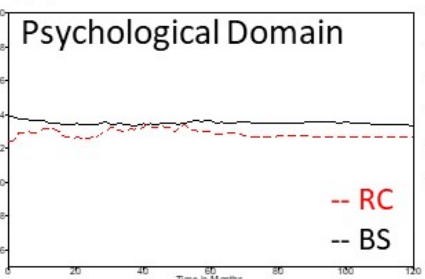

(F)

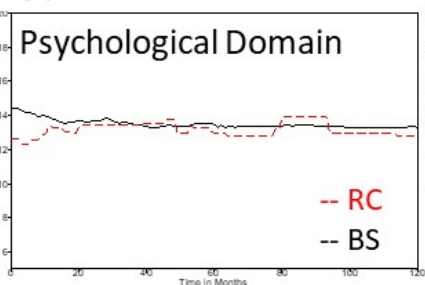

(J)

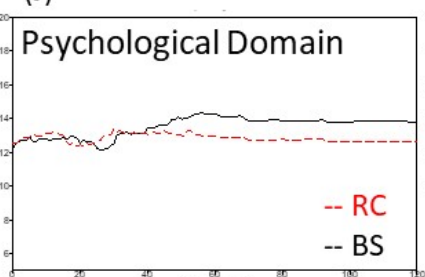

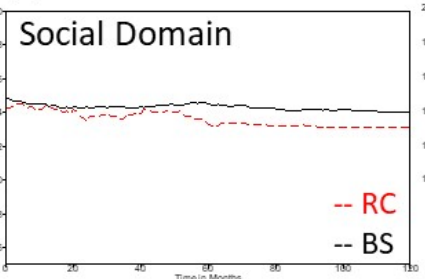

(G)

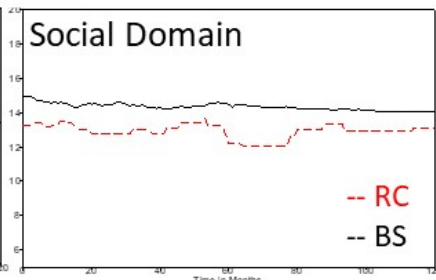

(K)

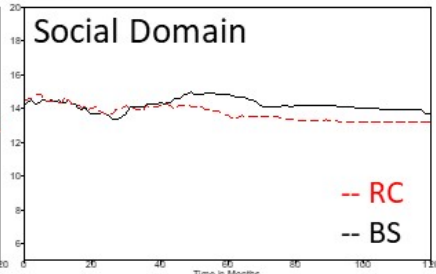

(D)

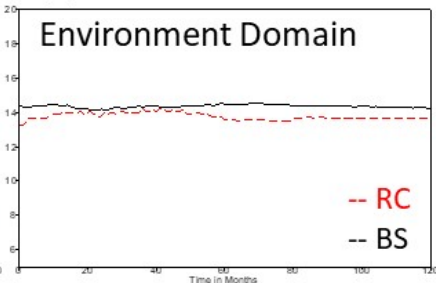

(H)

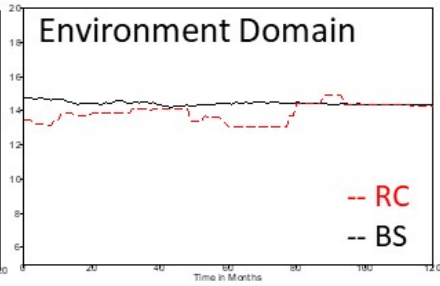

(L)

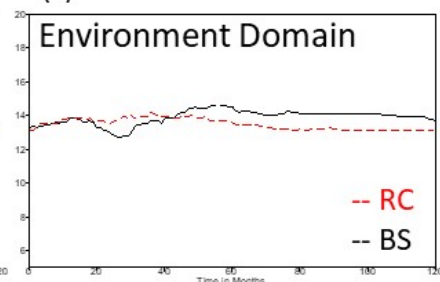

Figure 1. Dynamic changes in the scores for the four domains in the WHOQOL-BREF questionnaire depending on whether patients had or had not received a radical cystectomy. (A-D) all study subjects, (E-H) non-muscle-invasive bladder cancer patients, (I-L) patients with muscle-invasive bladder cancer or more severe conditions. WHOQOL-BREF, World Health Organization quality of life questionnaire brief version; RC, radical cystectomy; BS, bladder sparing.

\subsubsection{Item Score}

In the NMIBC subgroup, cystectomized patients had significantly lower scores for six items, including safety and security, physical environment, information acquiring, and home environment in the environment domain; activities of daily living in the physical domain; and sexual activity in the social domain, compared with patients who received bladder-sparing treatment. These differences occurred mainly in the first 40 months after the first definite treatment. Patients who received a radical cystectomy exhibited persistently lower scores for the sexual activity item in the social domain than those who received bladder-sparing treatment (Figure 2A). In the subgroup of patients with MIBC or more severe conditions, cystectomized patients had significantly lower scores for three items, including body image in the psychological domain and working capacity and sleep and rest in the physical domain. These differences became prominent from 40 months after the first definite treatment (Figure 2B). 
Table 2. Regression coefficients of the scores for each domain and item of the WHOQOL-BREF based on a mixed-effects model in NMIBC patients.

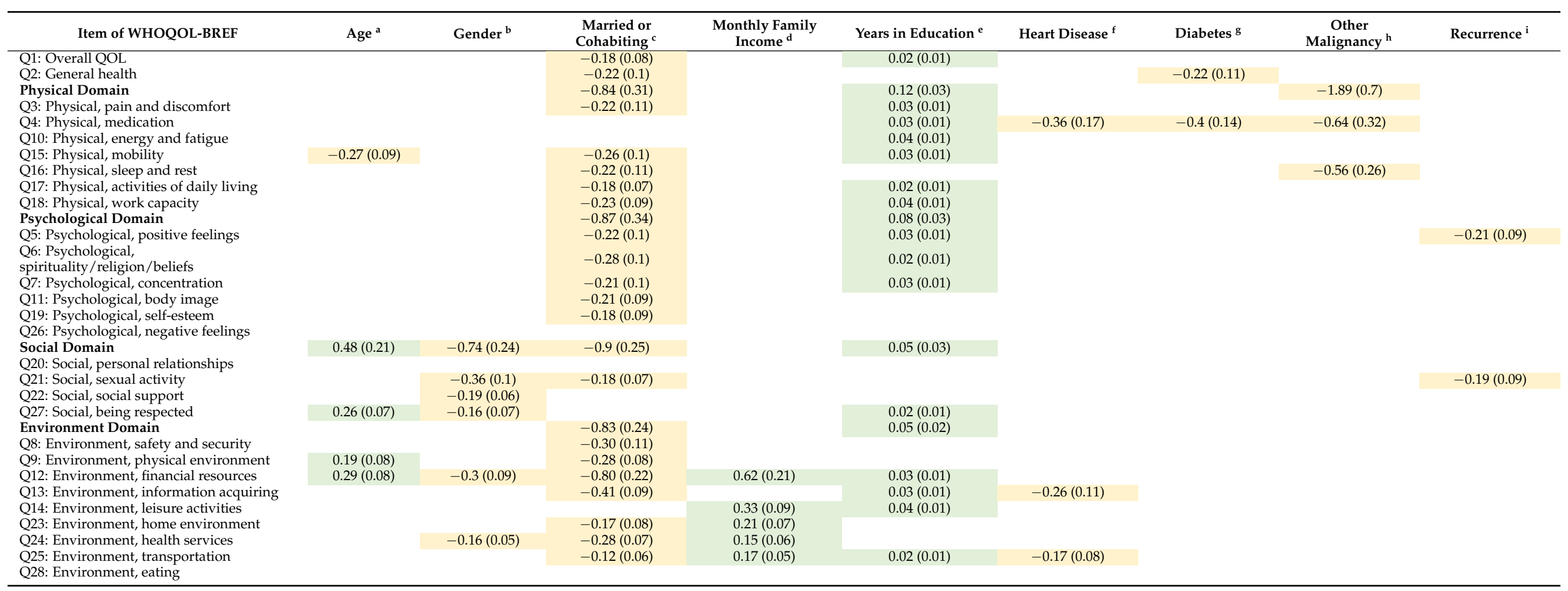

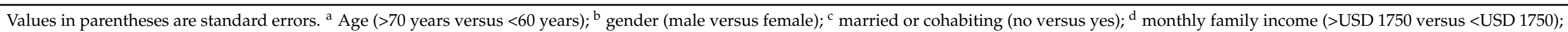

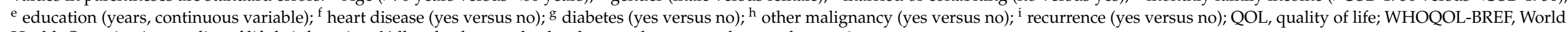
Health Organization quality of life brief version. Yellow background color denotes decrease and green denotes increase. 
Table 3. Regression coefficients of the scores for each domain and item of the WHOQOL-BREF based on a mixed-effects model in patients with MIBC or more severe conditions

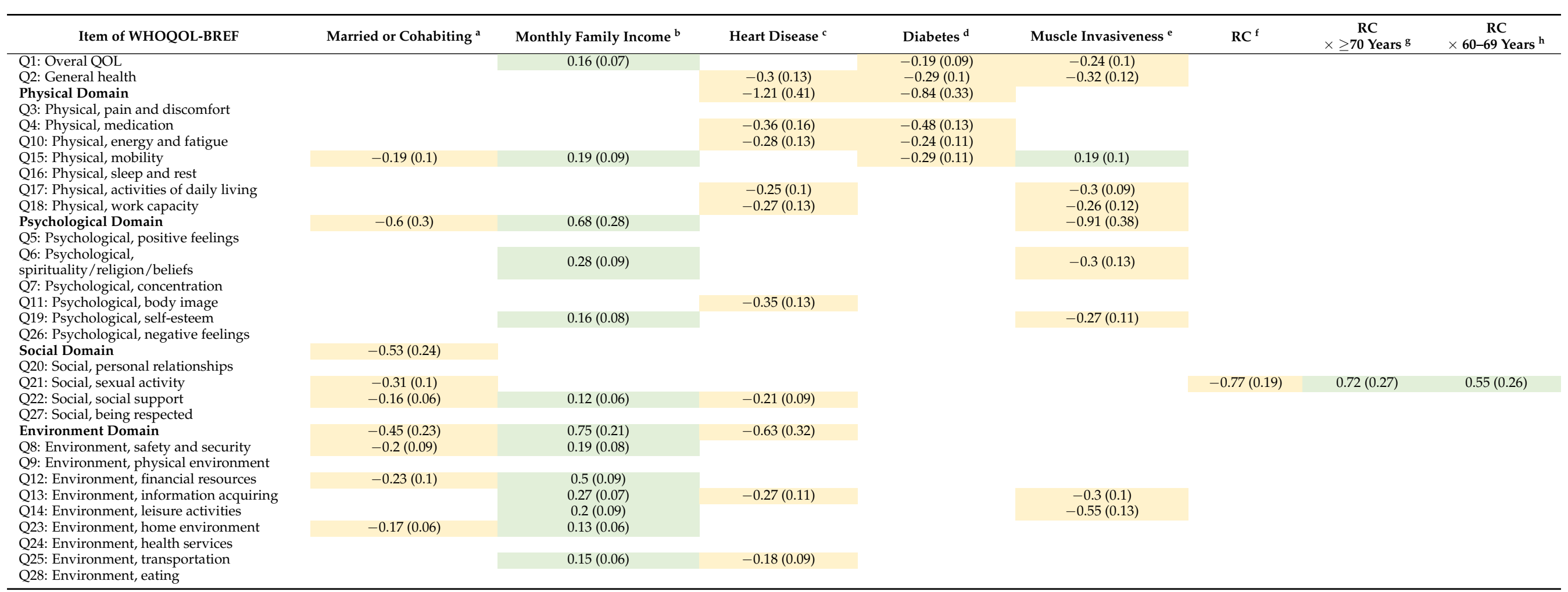

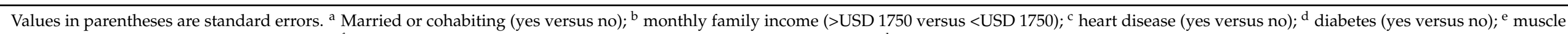

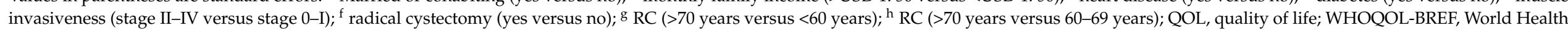
Organization quality of life brief version. Yellow background color denotes decrease and green denotes increase. 

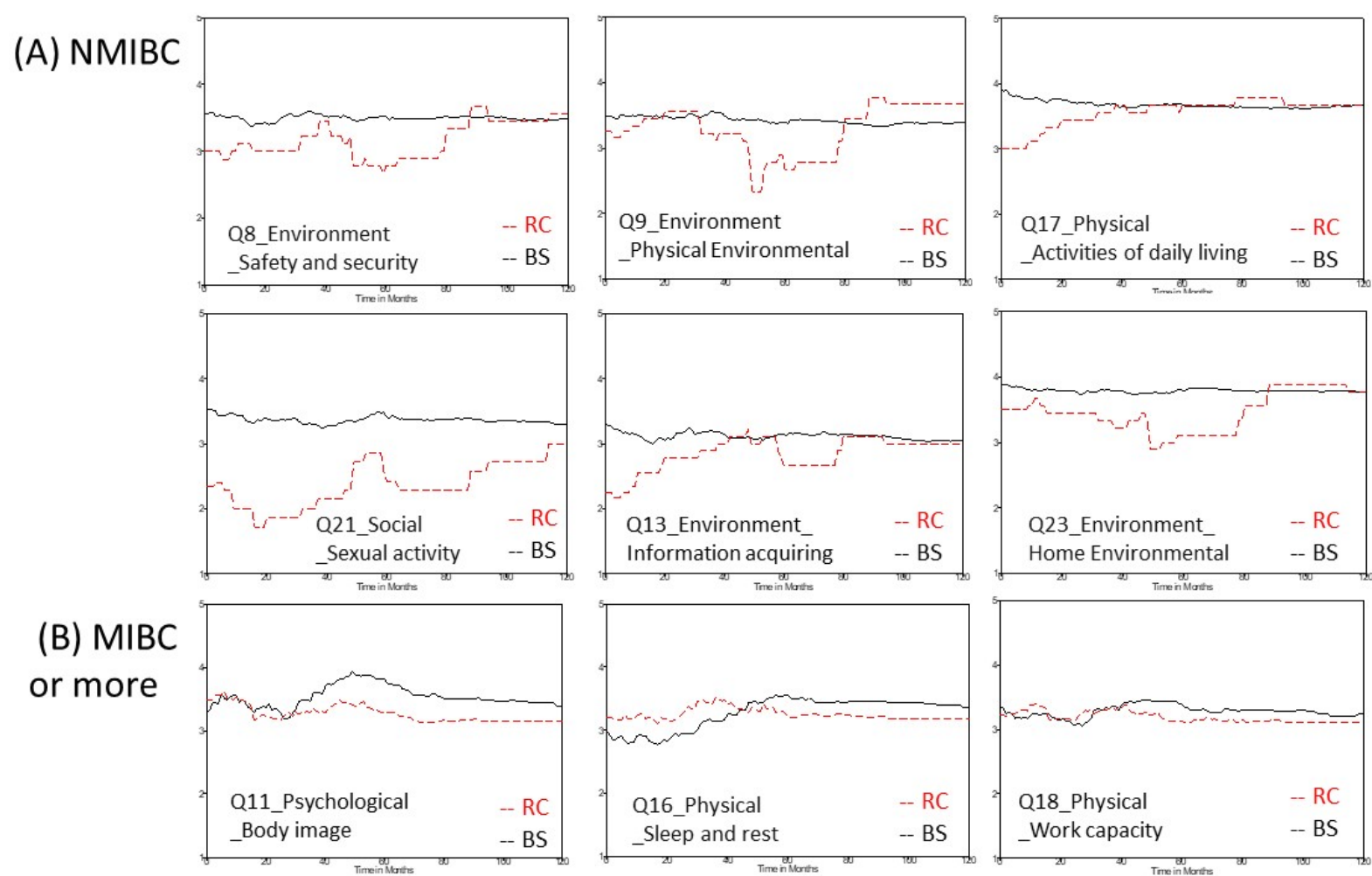

Figure 2. Dynamic changes in scores for certain items in the WHOQOL-BREF questionnaire according to whether or not patients received a radical cystectomy. (A) NIMBC patients, (B) patients with MIBC or more severe conditions. WHOQOLBREF, World Health Organization quality of life questionnaire brief version; RC, radical cystectomy; BS, bladder sparing; NMIBC, non-muscle-invasive bladder cancer; MIBC, muscle-invasive bladder cancer.

\section{Discussion}

Our study demonstrated the long-term dynamic changes in the QOL of patients with bladder cancer and their determinants. The results showed that cystectomized patients exhibited consistently lower QOL scores for each domain, starting from 40-50 months after the first definite treatment. Among them, NMIBC patients who received a cystectomy had worse QOL scores for the physical, psychological, and environment domains initially, but the differences compared with the patients with intact bladders disappeared 2 years after the first definite treatment, except for the persistently lower score for the social domain in cystectomized patients. The mixed-effects-model analysis also showed that both cystectomy and superficial bladder tumor recurrence had significant negative effects on the sexual activity item of the social domain. Most interestingly, the influence of a cystectomy on sexuality declined in patients older than 60 or 70 years, as shown in the areas of significant interaction for those aged over 60 years. These results highlight the importance of discussing private information such as sexuality before making a decision on treatment options.

This study collected long-term QOL data and comprehensively controlled for the potential confounding variables of different demographic and clinical factors through mixed-effects models. We found that being married or cohabiting, having a higher monthly family income, and having spent longer in education positively increased patients' QOL score, which corroborated many previous reports [27-31]. The negative effects of an advanced stage of cancer and comorbidity with heart disease and diabetes were also consistent with previous clinical observations [9,32]. In addition, younger patients' sexual activity was negatively affected by radical cystectomy [17,33]. In other words, these 
findings corroborated the validity of our models. Cystectomized patients may worry about the occasional foul odor from using diapers or urine bags and feel embarrassed while going out or participating in different social activities, which seems to have resulted in lower scores for most items in the physical and social domains, especially for the item of sexual activity. Not only can these determinants help clinicians to predict patients' satisfaction in terms of their QOL, but they also serve as an initial platform for shared decision making and help to optimize the value of different interventions.

Bladder cancer is a solid malignancy that has high cost burdens due to its high recurrence rates, intensive surveillance strategies, and expensive treatment costs [34]. After overcoming the risk of treatment morbidity and mortality, bladder cancer patients and their family members still have the heavy burden of non-medical expenditure. Our data demonstrated that certain factors influenced the scores for some items in the environment domain, including age, marital status, family income, years in education, and comorbidity with heart disease. In fact, patients must pay at least the following additional costs: transportation for each clinic visit, caregiver-time, urine collection bags and/or diapers, and productivity lost due to illness [35]. As there are so many items that are not included in the reimbursement schedule of our National Health Insurance system, these additional costs usually bring a heavy financial burden to patients with bladder cancer and affect almost every item and domain of their QOL questionnaire. Therefore, healthcare professionals must pay attention to these issues and make early referrals to social workers for access to social welfare and/or other support.

In this study, we found that heart disease and diabetes mellitus seemed to be more common in the non-cystectomy group. This might be because patients with heart disease and diabetes mellitus might be less able to undergo major surgery (radical cystectomy) and so choose to keep their bladder. We believe that our data reflect reality and could acceptably represent the population of patients with bladder cancer.

There are five limitations to this study. First, all participants were recruited from one medical center, which might limit the study's generalizability. However, as we included various types of patients and controlled for all demographic and clinical factors in our modeling, our conclusion regarding sexual activity would probably still be valid. Second, there may be bias between NMIBC patients and patients with MIBC or more severe conditions in terms of the number of patients who underwent a cystectomy. Therefore, we analyzed the QOL data in NMIBC patients who did not undergo a cystectomy, which may have reduced the bias in the NMIBC group. Although the number of NMIBC patients who underwent a radical cystectomy was small, NIMBC patients usually achieve long-term survival. Therefore, the long-term dynamic changes, as shown in Figure 1E-H and Figure 2A, can still provide some useful information in terms of generic QOL. Third, we did not categorize cystectomized patients into subgroups according to the accompanying diversion type, due to the limited sample size. Fourth, there were only four patients who received a radical cystectomy without any urinary diversion. All of them had ESRD and were on dialysis. Indeed, dialysis may influence QOL negatively. In fact, not only dialysis but also other factors (such as chemotherapy, radiotherapy, subsequent secondary malignancy, or other comorbidities) can greatly impact certain aspects of the generic QOL. Therefore, we used a mixed-effects model to reduce the effects from these confounders. Lastly, it seems inappropriate to make comparisons between non-metastatic MIBC patients who received a cystectomy and metastatic patients who did not receive a cystectomy owing to having unresectable tumors. However, some metastatic patients who responded well to initial systemic chemotherapy received a radical cystectomy and diversion and showed favorable outcomes because of the pathological downstaging response. Similarly, the mixed-effectsmodel method we used can reduce this bias after controlling for these confounders.

\section{Conclusions}

In this study, we demonstrated the long-term QOL changes in human bladder cancer patients according to a generic questionnaire. In general, marital status, monthly income, 
and comorbidity with heart disease and diabetes are significant determinants. Regardless of the urinary diversion type, radical cystectomy contributes to lower scores for all four domains, mainly from 4 to 5 years after the cystectomy, which declines significantly in patients older than 60 years. As for NMIBC patients with preserved bladders, tumor recurrence is a major predictor for lower scores for sexual activity in the social domain. These data reflect the general concerns of bladder cancer patients after the first definite treatment, which should be discussed with patients before deciding on the treatment selection.

Supplementary Materials: The following are available online at https: / www.mdpi.com/article / $10.3390 /$ jcm10235472/s1, Table S1: Comparison of demographics characteristics of bladder cancer patients according to number of interviews.

Author Contributions: Y.-S.T., T.-Y.W., and Y.-C.J.: Manuscript writing/editing; T.-S.T.: Data collection or management; Y.-S.T.: Data analysis and Manuscript writing/editing; J.-D.W.: Data analysis and Manuscript writing/editing. All authors have read and agreed to the published version of the manuscript.

Funding: The project has been under the long-term funding support of Oncology Center Grant of the Ministry of Health and Welfare (DOH102-TD-C-111-003, DOH102-TD-C-111-004, CA-103-SP-01, MOHW103-TD-B-111-06) and NCKUH (NCKUH-10206002).

Institutional Review Board Statement: The study began after obtaining written ethics committee approval from the Institutional Review Board of University Hospital of NCKU (A-ER-101-219). All the methods were performed following the rules of the Declaration of Helsinki.

Informed Consent Statement: All study subjects gave their informed consent for inclusion before they participated in the study.

Data Availability Statement: The clinical data, including age at diagnosis, pathological stage, date and types of surgery, periods of systemic chemotherapy and radiotherapy, were abstracted from electronic medical records of University Hospital of NCKU. The collected questionnaires were obtained from clinical cancer center of University Hospital. These data are not public and only available after obtaining the IRB consent.

Acknowledgments: We are grateful to all the patients who provided their quality of life data to this study. We are also indebted to Fat-Ya Ou. Without her consistent support in recruiting patients, this study would not have been completed.

Conflicts of Interest: The authors declare no conflict of interest. The funders had no role in the design of the study; in the collection, analyses, or interpretation of data; in the writing of the manuscript, or in the decision to publish the results.

\section{References}

1. Antoni, S.; Ferlay, J.; Soerjomataram, I.; Znaor, A.; Jemal, A.; Bray, F. Bladder cancer incidence and mortality: A global overview and recent trends. Eur. Urol. 2017, 71, 96-108. [CrossRef] [PubMed]

2. Chen, C.J.; Chuang, Y.C.; Lin, T.M.; Wu, H.Y. Malignant neoplasms among residents of a blackfoot disease-endemic area in Taiwan: High-arsenic artesian well water and cancers. Cancer Res. 1985, 45, 5895-5899. [PubMed]

3. Chen, C.H.; Grollman, A.P.; Huang, C.Y.; Shun, C.T.; Sidorenko, V.S.; Hashimoto, K.; Moriya, M.; Turesky, R.J.; Yun, B.H.; Tsai, K.; et al. Additive effects of arsenic and aristolochic acid in chemical carcinogenesis of upper urinary tract urothelium. Cancer Epidemiol. Prev. Biomark. 2021, 30, 317-325. [CrossRef] [PubMed]

4. Gerharz, E.W.; Mansson, A.; Hunt, S.; Skinner, E.C.; Mansson, W. Quality of life after cystectomy and urinary diversion: An evidence based analysis. J. Urol. 2005, 174, 1729-1736. [CrossRef]

5. Babjuk, M.; Burger, M.; Capoun, O.; Cohen, D.; Comperat, E.M.; Dominguez Escrig, J.L.; Gontero, P.; Liedberg, F.; MassonLecomte, A.; Mostafid, A.H.; et al. European association of urology guidelines on non-muscle-invasive bladder cancer (Ta, T1, and carcinoma in situ). Eur. Urol. 2021. [CrossRef]

6. Witjes, J.A.; Lebret, T.; Compérat, E.M.; Cowan, N.C.; De Santis, M.; Bruins, H.M.; Hernández, V.; Espinós, E.L.; Dunn, J.; Rouanne, M.; et al. Updated 2016 EAU guidelines on muscle-invasive and metastatic bladder cancer. Eur. Urol. 2017, 71, 462-475. [CrossRef] 
7. Powles, T.; Csoszi, T.; Ozguroglu, M.; Matsubara, N.; Geczi, L.; Cheng, S.Y.; Fradet, Y.; Oudard, S.; Vulsteke, C.; Morales Barrera, R.; et al. Pembrolizumab alone or combined with chemotherapy versus chemotherapy as first-line therapy for advanced urothelial carcinoma (KEYNOTE-361): A randomised, open-label, phase 3 trial. Lancet Oncol. 2021, 22, 931-945. [CrossRef]

8. Necchi, A.; Anichini, A.; Raggi, D.; Briganti, A.; Massa, S.; Luciano, R.; Colecchia, M.; Giannatempo, P.; Mortarini, R.; Bianchi, M.; et al. Pembrolizumab as neoadjuvant therapy before radical cystectomy in patients with muscle-invasive urothelial bladder carcinoma (PURE-01): An open-label, single-arm, phase II study. J. Clin. Oncol. 2018, 36, 3353-3360. [CrossRef]

9. Fung, C.; Pandya, C.; Guancial, E.; Noyes, K.; Sahasrabudhe, D.M.; Messing, E.M.; Mohile, S.G. Impact of bladder cancer on health related quality of life in 1,476 older Americans: A cross-sectional study. J. Urol. 2014, 192, 690-695. [CrossRef]

10. Ritch, C.R.; Cookson, M.S.; Chang, S.S.; Clark, P.E.; Resnick, M.J.; Penson, D.F.; Smith, J.A.; Jr May, A.T.; Anderson, C.B.; You, C.; et al. Impact of complications and hospital-free days on health related quality of life 1 year after radical cystectomy. $J$. Urol. 2014, 192, 1360-1364. [CrossRef]

11. Huang, Y.; Pan, X.; Zhou, Q.; Huang, H.; Li, L.; Cui, X.; Wang, G.; Jizhong, R.; Yin, L.; Xu, D.; et al. Quality-of-life outcomes and unmet needs between ileal conduit and orthotopic ileal neobladder after radical cystectomy in a Chinese population: A 2-to-1 matched-pair analysis. BMC Urol. 2015, 15, 117. [CrossRef]

12. Allareddy, V.; Kennedy, J.; West, M.M.; Konety, B.R. Quality of life in long-term survivors of bladder cancer. Cancer 2006, 106, 2355-2362. [CrossRef]

13. Gacci, M.; Saleh, O.; Cai, T.; Gore, J.L.; D’Elia, C.; Minervini, A.; Masieri, L.; Giannessi, C.; Lanciotti, M.; Varca, V.; et al. Quality of life in women undergoing urinary diversion for bladder cancer: Results of a multicenter study among long-term disease-free survivors. Health Qual. Life Outcomes 2013, 11, 43. [CrossRef]

14. Goldberg, H.; Baniel, J.; Mano, R.; Rotlevy, G.; Kedar, D.; Yossepowitch, O. Orthotopic neobladder vs. ileal conduit urinary diversion: A long-term quality-of-life comparison. Urol. Oncol. 2016, 34, 121.e1-121.e7. [CrossRef]

15. Kikuchi, E.; Horiguchi, Y.; Nakashima, J.; Ohigashi, T.; Oya, M.; Nakagawa, K.; Miyajima, A.; Murai, M. Assessment of long-term quality of life using the FACT-BL questionnaire in patients with an ileal conduit, continent reservoir, or orthotopic neobladder. Jpn. J. Clin. Oncol. 2006, 36, 712-716. [CrossRef]

16. Matsuda, T.; Aptel, I.; Exbrayat, C.; Grosclaude, P. Determinants of quality of life of bladder cancer survivors five years after treatment in France. Int. J. Urol. 2003, 10, 423-429. [CrossRef]

17. Mischinger, J.; Abdelhafez, M.F.; Todenhofer, T.; Schwentner, C.; Aufderklamm, S.; Stenzl, A.; Gakis, G. Quality of life outcomes after radical cystectomy: Long-term standardized assessment of Studer Pouch versus I-Pouch. World J. Urol. 2015, 33, 1381-1387. [CrossRef]

18. Rouanne, M.; Legrand, G.; Neuzillet, Y.; Ghoneim, T.; Cour, F.; Letang, N.; Yonneau, L.; Herve, J.M.; Botto, H.; Lebret, T. Long-term women-reported quality of life after radical cystectomy and orthotopic ileal neobladder reconstruction. Ann. Surg. Oncol. 2014, 21, 1398-1404. [CrossRef]

19. Takenaka, A.; Hara, I.; Soga, H.; Sakai, I.; Terakawa, T.; Muramaki, M.; Miyake, H.; Tanaka, K.; Fujisawa, M. Assessment of long-term quality of life in patients with orthotopic neobladder followed for more than 5 years. Int. Urol. Nephrol. 2011, 43, 749-754. [CrossRef]

20. Wright, J.L.; Porter, M.P. Quality-of-life assessment in patients with bladder cancer. Nat. Clin. Pract. Urol. 2007, 4, 147-154. [CrossRef]

21. Chung, J.; Kulkarni, G.S.; Morash, R.; Matthew, A.; Papadakos, J.; Breau, R.H.; Guttman, D.; Bender, J.; Jones, J.M. Assessment of quality of life, information, and supportive care needs in patients with muscle and non-muscle invasive bladder cancer across the illness trajectory. Support Care Cancer 2019, 27, 3877-3885. [CrossRef] [PubMed]

22. Harper, A. Development of the World Health Organisation WHOQOL-BREF quality of life assessment. Psychol. Med. 1998, 28, 551-558.

23. Yao, G.; Chung, C.W.; Yu, C.F.; Wang, J.D. Development and verification of validity and reliability of the WHOQOL-BREF Taiwan version. J. Formos. Med Assoc. Taiwan Yi Zhi 2002, 101, 342-351. [PubMed]

24. Kao, Y.L.; Ou, C.H.; Lin, S.H.; Chang, S.M.; Wang, J.D.; Tsai, Y.S. Dynamic changes of generic quality of life after different treatments for localized prostate cancer. J. Clin. Med. 2021, 10, 158. [CrossRef]

25. Wu, T.Y.; Chang, T.W.; Chang, S.M.; Lin, Y.Y.; Wang, J.D.; Kuo, Y.L. Dynamic changes of body image and quality of life in breast cancer patients. Cancer Manag. Res. 2019, 11, 10563-10571. [CrossRef]

26. iSQoL: Integration of Survival with Quality of Life. Available online: http://www.stat.sinica.edu.tw/isqol/ (accessed on 19 March 2019).

27. Datta, G.D.; Neville, B.A.; Kawachi, I.; Datta, N.S.; Earle, C.C. Marital status and survival following bladder cancer. J. Epidemiol. Community Health 2009, 63, 807-813. [CrossRef]

28. Gore, J.L.; Kwan, L.; Saigal, C.S.; Litwin, M.S. Marriage and mortality in bladder carcinoma. Cancer 2005, 104, 1188-1194. [CrossRef]

29. Nelles, J.L.; Joseph, S.A.; Konety, B.R. The impact of marriage on bladder cancer mortality. Urol. Oncol. 2009, 27, 263-267. [CrossRef]

30. Pruthi, R.S.; Lentz, A.C.; Sand, M.; Kouba, E.; Wallen, E.M. Impact of marital status in patients undergoing radical cystectomy for bladder cancer. World J. Urol. 2009, 27, 573-576. [CrossRef] 
31. Sammon, J.D.; Morgan, M.; Djahangirian, O.; Trinh, Q.D.; Sun, M.; Ghani, K.R.; Jeong, W.; Jhaveri, J.; Ehlert, M.; Schmitges, J.; et al. Marital status: A gender-independent risk factor for poorer survival after radical cystectomy. BJU Int. 2012, 110, 1301-1309. [CrossRef]

32. Cerruto, M.A.; D’Elia, C.; Siracusano, S.; Gedeshi, X.; Mariotto, A.; Iafrate, M.; Niero, M.; Lonardi, C.; Bassi, P.; Belgrano, E.; et al. Systematic review and meta-analysis of non RCT's on health related quality of life after radical cystectomy using validated questionnaires: Better results with orthotopic neobladder versus ileal conduit. Eur. J. Surg. Oncol. 2016, 42, 343-360. [CrossRef]

33. Modh, R.A.; Mulhall, J.P.; Gilbert, S.M. Sexual dysfunction after cystectomy and urinary diversion. Nat. Rev. Urol. 2014, 11, 445-453. [CrossRef]

34. Svatek, R.S.; Hollenbeck, B.K.; Holmang, S.; Lee, R.; Kim, S.P.; Stenzl, A.; Lotan, Y. The economics of bladder cancer: Costs and considerations of caring for this disease. Eur. Urol. 2014, 66, 253-262. [CrossRef]

35. Sanders, G.D.; Neumann, P.J.; Basu, A.; Brock, D.W.; Feeny, D.; Krahn, M.; Kuntz, K.M.; Meltzer, D.O.; Owens, D.K.; Prosser, L.A.; et al. Recommendations for conduct, methodological practices, and reporting of cost-effectiveness analyses: Second panel on cost-effectiveness in health and medicine. JAMA 2016, 316, 1093-1103. [CrossRef] 